Xiang Yu, You-Fang Zhao, Yan Qin, Jing Yan and Ya-Fang Chen*

\title{
The crystal structure of $N^{\prime}-((1 E, 2 E)-4-(7-m e t h o x y-$ 2-oxo-2H-chromen-8-yl)-2-methylbut-2-en-1- ylidene)-3-methylbenzohydrazide, $\mathrm{C}_{23} \mathrm{H}_{22} \mathrm{~N}_{2} \mathrm{O}_{4}$
}

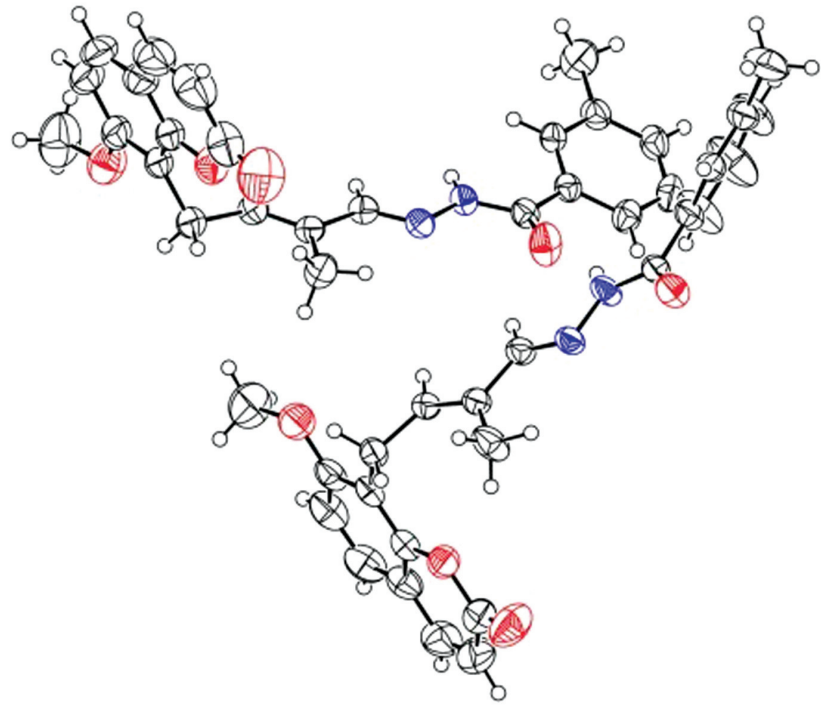

https://doi.org/10.1515/ncrs-2019-0249

Received April 7, 2019; accepted June 13, 2019; available online June 22, 2019

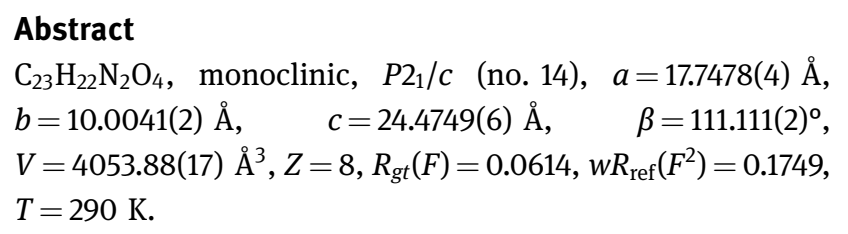

CCDC no.: 1922778

The asymmetric unit containing two crystallographically independent molecules is shown in the figure. Table 1 contains crystallographic data and Table 2 contains the list of the atoms including atomic coordinates and displacement parameters.

\footnotetext{
*Corresponding author: Ya-Fang Chen, Guizhou University of Traditional Chinese Medicine, Guiyang 550025, P.R. China, e-mail: 985566851@qq.com

Xiang Yu, You-Fang Zhao, Yan Qin and Jing Yan: Guizhou University of Traditional Chinese Medicine, Guiyang 550025, P.R. China
}

Ә Open Access. ( 2019 Xiang Yu et al., published by De Gruyter. (cc) BY License.
Table 1: Data collection and handling.

\begin{tabular}{ll}
\hline Crystal: & Colourless block \\
Size: & $0.29 \times 0.28 \times 0.27 \mathrm{~mm}$ \\
Wavelength: & Mo $K \alpha$ radiation $(0.71073 \AA)$ \\
$\mu:$ & $0.09 \mathrm{~mm}^{-1}$ \\
Diffractometer, scan mode: & SuperNova, $\omega$ \\
$\theta_{\text {max }}$, completeness: & $28.5^{\circ},>99 \%$ \\
$N(h k l)_{\text {measured }}, N(h k l)_{\text {unique }}, R_{\text {int }}:$ & $93062,9585,0.055$ \\
Criterion for $I_{\text {obs }}, N\left(h k l_{\text {gt }}:\right.$ & $I_{\text {obs }}>2 \sigma\left(I_{\text {obs }}\right), 6513$ \\
$N(\text { param })_{\text {refined }}:$ & 529 \\
Programs: & CrysAlis \\
& SHEL $[1]$, Olex2 [2], \\
\hline
\end{tabular}

\section{Source of material}

In a representative experiment 7-methoxy-8-(3-methylbut2-en-1-yl)-2H-chromen-2-one $(1.0 \mathrm{mmol})$ reacted with $\mathrm{SeO}_{2}$ $(1.1 \mathrm{mmol})$ in dioxane $(10 \mathrm{~mL})$ at $80{ }^{\circ} \mathrm{C}$ to afford the aldehyde, and then reacted with 4-methylbenzoyl hydrazine $(1.0 \mathrm{mmol})$, and two drops of $\mathrm{HOAc}$ in $\mathrm{EtOH}(10 \mathrm{~mL})$ at $80{ }^{\circ} \mathrm{C}$. Suitable colorless block crystals of this compound were then obtained after recrystallization from methanol.

\section{Experimental details}

The structure was solved with the Olex2 program [2] as an interface together with the SHELXT and SHELXL programs $[3,4]$. All non-hydrogen atoms were assigned anisotropic displacement parameters in the refinement. Hydrogen atoms were treated using a riding model.

\section{Comment}

Coumarins were natural products with diverse bioactivities such as anti-tumor [5], anti-inflammatory [6] and antimicrobial activity [7]. On the other hand, recent studies show that introduction of hydrazone fragments into natural products could displayed the remarkable biological activity [8, 9]. In order to discover more compounds with potential bioactivities we prepared a coumarin-based hydrazone derivative by introduction of hydrazone moiety into the coumarin.

The asymmetric unit of the title structure consists of two crystallographically independent molecules ( $c f$. the figure). Geometric parameters of the title structure are in the usual 
Table 2: Fractional atomic coordinates and isotropic or equivalent isotropic displacement parameters $\left(\AA^{2}\right)$.

\begin{tabular}{|c|c|c|c|c|}
\hline Atom & $x$ & $y$ & $z$ & $U_{\text {iso }} * / U_{\text {eq }}$ \\
\hline 01 & $0.26905(9)$ & $0.96583(15)$ & $0.33980(6)$ & $0.0499(4)$ \\
\hline 02 & $0.24264(14)$ & $1.1723(2)$ & $0.30743(9)$ & $0.0881(6)$ \\
\hline 03 & $0.34233(11)$ & $0.54040(16)$ & $0.42024(8)$ & $0.0645(5)$ \\
\hline 04 & $0.16120(10)$ & $1.24119(13)$ & $0.61106(7)$ & $0.0517(4)$ \\
\hline N1 & $0.20741(10)$ & $1.05625(16)$ & $0.54586(7)$ & $0.0422(4)$ \\
\hline N2 & $0.15460(11)$ & $1.03074(16)$ & $0.57499(8)$ & $0.0448(4)$ \\
\hline $\mathrm{H} 2$ & 0.1341 & 0.9522 & 0.5731 & $0.054^{*}$ \\
\hline $\mathrm{C} 1$ & $0.23379(15)$ & $1.0559(3)$ & $0.29467(11)$ & $0.0613(6)$ \\
\hline C2 & $0.19009(17)$ & $1.0006(3)$ & $0.23797(12)$ & $0.0736(8)$ \\
\hline $\mathrm{H} 2 \mathrm{~A}$ & 0.1672 & 1.0577 & 0.2063 & $0.088^{\star}$ \\
\hline C3 & $0.18169(16)$ & $0.8688(3)$ & $0.22975(11)$ & $0.0708(7)$ \\
\hline H3 & 0.1515 & 0.8360 & 0.1928 & $0.085^{\star}$ \\
\hline $\mathrm{C} 4$ & $0.21820(14)$ & $0.7768(3)$ & $0.27665(10)$ & $0.0534(6)$ \\
\hline C5 & $0.21527(16)$ & $0.6387(3)$ & $0.27125(12)$ & $0.0653(7)$ \\
\hline H5 & 0.1853 & 0.6008 & 0.2353 & $0.078^{\star}$ \\
\hline C6 & $0.25518(16)$ & $0.5564(3)$ & $0.31733(12)$ & $0.0626(7)$ \\
\hline H6 & 0.2522 & 0.4641 & 0.3127 & $0.075^{\star}$ \\
\hline C7 & $0.30073(14)$ & $0.6131(2)$ & $0.37172(10)$ & $0.0499(5)$ \\
\hline C8 & $0.30533(12)$ & $0.7516(2)$ & $0.37966(9)$ & $0.0407(5)$ \\
\hline C9 & $0.26371(12)$ & $0.8292(2)$ & $0.33160(9)$ & $0.0429(5)$ \\
\hline C10 & $0.3495(2)$ & $0.3997(3)$ & $0.41407(17)$ & $0.0938(10)$ \\
\hline $\mathrm{H} 10 \mathrm{~A}$ & 0.3751 & 0.3827 & 0.3862 & $0.141^{*}$ \\
\hline $\mathrm{H} 10 \mathrm{~B}$ & 0.2968 & 0.3598 & 0.4006 & $0.141^{*}$ \\
\hline $\mathrm{H} 10 \mathrm{C}$ & 0.3815 & 0.3619 & 0.4513 & $0.141^{*}$ \\
\hline C11 & $0.35239(12)$ & $0.8148(2)$ & $0.43816(9)$ & $0.0415(4)$ \\
\hline $\mathrm{H} 11 \mathrm{~A}$ & 0.3963 & 0.7561 & 0.4602 & 0.050 * \\
\hline H11B & 0.3755 & 0.8986 & 0.4319 & $0.050^{\star}$ \\
\hline C12 & $0.29926(11)$ & $0.84007(19)$ & $0.47269(8)$ & $0.0379(4)$ \\
\hline H12 & 0.2756 & 0.7650 & 0.4822 & $0.045^{\star}$ \\
\hline C13 & $0.28159(11)$ & $0.95671(19)$ & $0.49148(8)$ & $0.0380(4)$ \\
\hline C14 & $0.22740(12)$ & $0.9522(2)$ & $0.52403(9)$ & $0.0402(4)$ \\
\hline H14 & 0.2064 & 0.8700 & 0.5292 & $0.048^{\star}$ \\
\hline C15 & $0.31369(16)$ & $1.0894(2)$ & $0.48191(12)$ & $0.0609(6)$ \\
\hline H15A & 0.3712 & 1.0912 & 0.5018 & 0.091 * \\
\hline H15B & 0.2895 & 1.1589 & 0.4971 & $0.091^{*}$ \\
\hline H15C & 0.3010 & 1.1031 & 0.4407 & $0.091^{*}$ \\
\hline C16 & $0.13491(12)$ & $1.12703(19)$ & $0.60623(9)$ & $0.0398(4)$ \\
\hline C17 & $0.07825(13)$ & $1.08329(19)$ & $0.63543(9)$ & $0.0439(5)$ \\
\hline C18 & $0.09078(14)$ & $1.1311(2)$ & $0.69118(9)$ & $0.0458(5)$ \\
\hline H18 & 0.1341 & 1.1879 & 0.7094 & $0.055^{\star}$ \\
\hline C19 & $0.03929(16)$ & $1.0952(2)$ & $0.72021(11)$ & $0.0569(6)$ \\
\hline $\mathrm{C} 20$ & $-0.0245(2)$ & $1.0119(3)$ & $0.69171(15)$ & $0.0821(10)$ \\
\hline $\mathrm{H} 20$ & -0.0594 & 0.9869 & 0.7106 & $0.099^{\star}$ \\
\hline C21 & $-0.0380(2)$ & $0.9649(3)$ & $0.63670(17)$ & $0.0982(12)$ \\
\hline $\mathrm{H} 21$ & -0.0819 & 0.9095 & 0.6184 & $0.118^{\star}$ \\
\hline C22 & $0.01351(18)$ & $0.9998(3)$ & $0.60823(14)$ & $0.0771(9)$ \\
\hline H22 & 0.0047 & 0.9671 & 0.5709 & $0.093^{*}$ \\
\hline C23 & $0.0528(2)$ & $1.1483(4)$ & $0.78048(13)$ & $0.0904(10)$ \\
\hline $\mathrm{H} 23 \mathrm{~A}$ & 0.1056 & 1.1874 & 0.7966 & $0.136^{*}$ \\
\hline $\mathrm{H} 23 \mathrm{~B}$ & 0.0486 & 1.0764 & 0.8052 & $0.136^{*}$ \\
\hline $\mathrm{H} 23 \mathrm{C}$ & 0.0128 & 1.2149 & 0.7781 & $0.136^{*}$ \\
\hline 05 & $0.56386(10)$ & $0.46774(18)$ & $0.70392(8)$ & $0.0614(4)$ \\
\hline 06 & $0.59196(16)$ & $0.6733(3)$ & $0.73497(13)$ & $0.1088(8)$ \\
\hline 07 & $0.50174(11)$ & $0.04222(18)$ & $0.62100(9)$ & $0.0744(5)$ \\
\hline 08 & $0.11940(10)$ & $0.74961(14)$ & $0.56374(9)$ & $0.0658(5)$ \\
\hline N3 & $0.23454(9)$ & $0.55897(16)$ & $0.58128(7)$ & $0.0373(4)$ \\
\hline
\end{tabular}

Table 2 (continued)

\begin{tabular}{|c|c|c|c|c|}
\hline Atom & $x$ & $y$ & $z$ & $\boldsymbol{U}_{\text {iso }} * / \boldsymbol{U}_{\text {eq }}$ \\
\hline N4 & $0.15676(9)$ & $0.53398(15)$ & $0.57946(7)$ & $0.0366(4)$ \\
\hline H4 & 0.1431 & 0.4539 & 0.5847 & $0.044^{\star}$ \\
\hline $\mathrm{C} 24$ & $0.60162(18)$ & $0.5575(4)$ & $0.74803(15)$ & $0.0775(8)$ \\
\hline $\mathrm{C} 25$ & $0.6489(2)$ & $0.5020(4)$ & $0.80416(15)$ & $0.0917(10)$ \\
\hline H25 & 0.6753 & 0.5593 & 0.8351 & $0.110^{\star}$ \\
\hline $\mathrm{C} 26$ & $0.65588(19)$ & $0.3688(4)$ & $0.81295(13)$ & $0.0879(10)$ \\
\hline H26 & 0.6870 & 0.3360 & 0.8497 & $0.106^{\star}$ \\
\hline $\mathrm{C} 27$ & $0.61669(14)$ & $0.2782(3)$ & $0.76716(11)$ & $0.0656(7)$ \\
\hline $\mathrm{C} 28$ & $0.62150(16)$ & $0.1388(3)$ & $0.77156(13)$ & $0.0756(9)$ \\
\hline $\mathrm{H} 28$ & 0.6511 & 0.1004 & 0.8075 & $0.091^{\star}$ \\
\hline C29 & $0.58408(16)$ & $0.0571(3)$ & $0.72479(14)$ & $0.0710(8)$ \\
\hline H29 & 0.5879 & -0.0353 & 0.7291 & $0.085^{\star}$ \\
\hline C30 & $0.54028(13)$ & $0.1141(3)$ & $0.67072(12)$ & $0.0570(6)$ \\
\hline C31 & $0.53350(12)$ & $0.2530(2)$ & $0.66384(10)$ & $0.0477(5)$ \\
\hline C32 & $0.57150(13)$ & $0.3314(3)$ & $0.71235(11)$ & $0.0531(6)$ \\
\hline C33 & $0.5124(2)$ & $-0.0993(3)$ & $0.62216(19)$ & $0.1038(12)$ \\
\hline $\mathrm{H} 33 \mathrm{~A}$ & 0.4833 & -0.1354 & 0.5840 & $0.156^{\star}$ \\
\hline H33B & 0.4923 & -0.1379 & 0.6501 & $0.156^{*}$ \\
\hline $\mathrm{H} 33 \mathrm{C}$ & 0.5688 & -0.1196 & 0.6331 & $0.156^{\star}$ \\
\hline C34 & $0.48551(12)$ & $0.3149(2)$ & $0.60558(10)$ & $0.0490(5)$ \\
\hline $\mathrm{H} 34 \mathrm{~A}$ & 0.5104 & 0.3986 & 0.6013 & 0.059 * \\
\hline H34B & 0.4860 & 0.2556 & 0.5743 & $0.059^{\star}$ \\
\hline C35 & $0.40024(11)$ & $0.3401(2)$ & $0.60045(9)$ & $0.0419(5)$ \\
\hline H35 & 0.3710 & 0.2659 & 0.6044 & $0.050^{*}$ \\
\hline C36 & $0.36113(11)$ & $0.45670(19)$ & $0.59091(8)$ & $0.0370(4)$ \\
\hline C37 & $0.27756(11)$ & $0.45395(19)$ & $0.58787(8)$ & $0.0370(4)$ \\
\hline H37 & 0.2546 & 0.3720 & 0.5909 & $0.044^{\star}$ \\
\hline C38 & $0.39748(13)$ & $0.5875(2)$ & $0.58287(11)$ & $0.0551(6)$ \\
\hline $\mathrm{H} 38 \mathrm{~A}$ & 0.4054 & 0.5870 & 0.5461 & $0.083^{\star}$ \\
\hline H38B & 0.4485 & 0.5998 & 0.6141 & $0.083^{\star}$ \\
\hline $\mathrm{H} 38 \mathrm{C}$ & 0.3617 & 0.6592 & 0.5832 & $0.083^{*}$ \\
\hline C39 & $0.10250(12)$ & $0.63346(19)$ & $0.56957(9)$ & $0.0388(4)$ \\
\hline C40 & $0.01956(11)$ & $0.59498(19)$ & $0.56642(8)$ & $0.0365(4)$ \\
\hline C41 & $0.00208(11)$ & $0.4783(2)$ & $0.58977(9)$ & $0.0390(4)$ \\
\hline H41 & 0.0433 & 0.4174 & 0.6074 & $0.047^{\star}$ \\
\hline C42 & $-0.07599(12)$ & $0.4500(2)$ & $0.58743(9)$ & $0.0451(5)$ \\
\hline $\mathrm{C} 43$ & $-0.13603(12)$ & $0.5427(2)$ & $0.56054(10)$ & $0.0497(5)$ \\
\hline H43 & -0.1883 & 0.5264 & 0.5591 & $0.060^{*}$ \\
\hline C44 & $-0.12031(13)$ & $0.6574(2)$ & $0.53608(10)$ & $0.0515(6)$ \\
\hline H44 & -0.1618 & 0.7173 & 0.5178 & 0.062 * \\
\hline C45 & $-0.04303(12)$ & $0.6843(2)$ & $0.53851(9)$ & $0.0456(5)$ \\
\hline H45 & -0.0325 & 0.7619 & 0.5216 & $0.055^{\star}$ \\
\hline C46 & $-0.09364(15)$ & $0.3244(3)$ & $0.61374(14)$ & $0.0766(8)$ \\
\hline $\mathrm{H} 46 \mathrm{~A}$ & -0.0903 & 0.2492 & 0.5903 & $0.115^{\star}$ \\
\hline H46B & -0.1470 & 0.3292 & 0.6150 & $0.115^{\star}$ \\
\hline $\mathrm{H} 46 \mathrm{C}$ & -0.0550 & 0.3140 & 0.6528 & $0.115^{\star}$ \\
\hline
\end{tabular}

ranges [10]. The compound contains one methoxy group, one lactone and two methyl-groups. The length of $\mathrm{C}-\mathrm{C}$ double bond are, longer than the length of $\mathrm{C}-\mathrm{N}$ double bond. Both of the configuration of the $\mathrm{C}-\mathrm{C}$ double bond and the configuration $\mathrm{C}-\mathrm{N}$ double bond are in type $\mathrm{E}$, as well as the similar structure that reported [11]. 
Acknowledgements: This work was supported by the Science and Technology Fund of Guizhou [No.(2017)5735-22], the Youth Talent Development Project of Guizhou Provincial Department of Education [2017]169, and Special Topics for Scientific and Technological Research on Traditional Chinese Medicine and Ethnic Medicine [QZYY2017-081].

\section{References}

1. Oxford Diffraction. CrysAlis ${ }^{\text {Pro }}$ 1.171.39.46, SCALE3 ABSPACK, Oxford Diffraction Ltd, Yarnton, England (2018).

2. Dolomanov, O. V.; Bourhis, L. J.; Gildea, R. J.; Howard, J. A. K.; Puschmann, H.: OLEX2: a complete structure solution, refinement and analysis program. J. Appl. Crystallogr. 42 (2009) 339-341.

3. Sheldrick, G. M.: SHELXT - integrated space-group and crystalstructure determination. Acta Crystallogr. A71 (2015) 3-8.

4. Sheldrick, G. M.: Crystal structure refinement with SHELXL. Acta Crystallogr. C71 (2015) 3-8.

5. Kim, Y. K.; Kim, Y. S.; Ryu, S. Y.: Antiproliferative effect of furanocoumarins from the root of angelica on cultured human tumor cell lines. Phytother. Res. 3 (2007) 288-300.
6. Adad, M. J.; Delas, H. B.; Silvan, A. M.: Effects of furocoumarins from cachrys trifida on some macrophage functions. J. Pharm. Pharmacal. 8 (2001) 1163-1168.

7. Rosselli, S.; Maggio, A.; Bellone, G.: Antibacterial and anticoagulant activities of coumarins isolated from the flowers of Magydaris tomentosa. Planta Med. 2 (2007) 116-120.

8. Qu, H.; Lv, M.; Yu, X.; Lian, X. H.; Xu, H.: Discovery of some piperine-based phenylsulfonylhydrazone derivatives as potent botanically narcotic agents. Sci. Rep. 5 (2015) 13077.

9. Yu, X.; Feng, G.; Huang, J. L.; Xu, H.: Evaluation of some quinoline-based hydrazone derivatives as insecticidal agents. RSC Adv. 6 (2016) 30405-30411.

10. Yu, X.; Qin, Y.; Yan, J.; Chen, Y.-F.: Crystal structure of $\mathrm{N}^{\prime}-((1 E$, 2E)-4-(7-methoxy-2-oxo-2H-chromen-8-yl)-2-methylbut-2-en-1ylidene)-4-methylbenzenesulfonohydrazide, $\mathrm{C}_{22} \mathrm{H}_{22} \mathrm{O}_{5} \mathrm{~N}_{2} \mathrm{~S}$. Z. Kristallogr. NCS 234 (2019) 367-368.

11. Qu, H.; Yu, X.; Zhi, X. Y.; Lv, M.; Xu, H.: Natural-product-based insecticidal agents 14 . semisynthesis and insecticidal activity of new piperine-based hydrazone derivatives against Mythimna separata Walker in vivo. Bioorg. Med. Chem. Lett. 20 (2013) 5552-5557. 